\title{
Very Selective Detection of Low Physiopathological Glucose Levels by Spontaneous Raman Spectroscopy with Univariate Data Analysis
}

\author{
Ata Golparvar ${ }^{1}$ (D) $\cdot$ Assim Boukhayma ${ }^{1,2} \cdot$ Timothy Loayza $^{1} \cdot$ Antonino Caizzone $^{1,2} \cdot$ Christian Enz $^{1} \cdot$ Sandro Carrara $^{1}$
}

Accepted: 15 April 2021 / Published online: 8 May 2021

(C) The Author(s) 2021

\begin{abstract}
After decades of research on non-invasive glucose monitoring, invasive devices based on finger blood sampling are still the predominant reference for diabetic patients for accurately measuring blood glucose levels. Meanwhile, research continues improving point-of-care technology toward the development of painless and more accurate devices. Raman spectroscopy is well-known as a potentially valuable and painless approach for measuring glucose levels. However, previous Raman studies deal with glucose concentrations that are still order of magnitudes away with respect to human tissues' physiological concentrations, or they propose enhancement methodologies either invasive or much complex to assure sufficient sensitivity in the physiological range. Instead, this study proposes an alternative non-enhanced Raman spectroscopy approach sensitive to glucose concentrations from 1 to $5 \mathrm{mmol} / \mathrm{l}$, which correspond to the lowest physiopathological glucose level in human blood. Our findings suggest a very selective detection of glucose with respect to other typical metabolites, usually interfering with Raman spectroscopy's glucose detection. We validate the proposed univariate sensing methodology on glucose solutions mixed with lactate and urea, the two most common molecules found in human serum with concentrations similar to glucose and similar features in the Raman spectra. Our findings clearly illustrate that reliable detection of glucose by Raman spectroscopy is feasible by exploiting the shifted peak at $1125 \pm 10 \mathrm{~cm}^{-1}$ within physiopathological ranges.
\end{abstract}

Keywords Glucometer $\cdot$ Lactate sensing $\cdot$ Raman spectroscopy $\cdot$ Urea sensing $\cdot$ Non-invasive $\cdot$ Point-of-care

\section{Introduction}

At the beginning of the third decade of the twenty-first century, diabetes mellitus is a serious public health burden that seems to remain an exhaustive threat to human health in the years to come [1]. Diabetes is a chronic disorder that either impairs the pancreas to produce insulin or infects cells' insulin receptors to become desensitize or less sensitive to insulin and alleviates cells' capability to absorb glucose [2]. Therefore, diabetic patients suffer from the malfunction of glucose homeostasis, and to survive, they must prevent severe secondary complications. Consequently, careful "diabetes management" via frequent monitoring of blood glucose keeps the glucose

Ata Golparvar

ata.golparvar@epfl.ch

1 Integrated Circuit Laboratory (ICLAB), École Polytechnique Fédérale de Lausanne (EPFL), CH-2002 Neuchâtel, Switzerland

2 Senbiosys SA, CH-2002 Neuchâtel, Switzerland level under control through adequate insulin injection [3]. In fact, for nearly 150 to 200 million diabetics worldwide, controlling their glucose level is associated with daily exogenous insulin administration [4]. Moreover, glucose monitoring on multiple daily occasions is recommended, particularly in insulin-dependent therapies, since incorrect administration of insulin can be life-threatening [5]. Even if self-monitoring of blood glucose is less frequent for patients in non-insulin therapies [6], it is unavoidable to tailor their treatments to individuals, especially critical during pregnancy [7]. Although binding of glucose to proteins in the bloodstream presents vital elements in long-term glycemic markers, such as glycated hemoglobin (HbAlc) [8, 9], blood withdrawal-based (e.g., finger-prick) glucose level measurement through electrochemical reaction mechanisms remains the "gold standard" for both diagnosis and therapeutic decision-making [10].

However, despite the many benefits of finger-prick devices, several further implications are associated with the issue of finger sticking [11]. For instance, finger pricking is closely related to diabetes burnout (i.e., state of detachment from diabetic care) [12], which is directly related to diabetes-induced morbidity and mortality [13-15]. As a result, almost two 
decades ago, minimally invasive glucose monitoring devices, cumulatively known as continuous glucose monitoring devices, such as Dexcom (CA, USA), appeared on the glucose biosensor market. However, current continuous glucose monitoring devices have some drawbacks compared to fingerprick devices. They are challenging to use, may impose some degree of pain on the device's insertion, and need occasional finger pricking for calibration purposes [16]. More importantly, these products are costly, suffer an inherent response delay in "true" glucose level detection and lack accuracy for lower glucose concentration range, and are likely to miss hypoglycemia (i.e., a severe low blood glucose level requiring immediate treatment) [16]. These factors prohibit the substitution of standard finger-prick devices with state-of-the-art continuous glucose monitoring products. Therefore, the full potential of continuous painless monitoring is yet to be realized due to limitations in the principle of current technology's sensing mechanism [17]. New sensing approaches for non-invasive glucometers and numerous innovative designs have been investigated in the past, with varying degrees of success, for instance, GlucoWatch (Cygnus Inc., Idaho, USA) or the Pendra (Pendragon Medical Ltd., Bradford, UK) [18]. However, most of these approaches are susceptible to temperature changes and skin heterogeneity variations or produce irritations [18]. They generally come with bulky setups or have foreseen longtime measures and complicated calibrations [19]. Consequently, the unsatisfied need for better sensing of blood glucose levels has led to emerging techniques that capitalize on other painless optical sensing mechanisms.

Approaches based on Raman scattering offer novel solutions for label-free monitoring techniques [20], including glucose detection [21]. Raman spectroscopy is a class of analytical spectroscopic techniques based on the inelastic energy exchange with respect to the rotational and vibrational modes of the analyses' molecular structure. Typically, the Raman spectroscopy offers a unique chemical "fingerprint" signature of the measurand to be specified [20]. Despite the lower quantum efficiency of inelastic light scattering, Raman effectbased glucometers offer more robustness concerning absorption spectroscopy, thanks to the water's inadequate scattering response but high absorbance signature. Nevertheless, to increase its quantum efficacy and lower the acquisition time and required source power, methods such as surface enhancement and coherent Raman scattering have been suggested [20]. Although studies based on surface enhancement demonstrated promising results even for in vivo monitoring of blood glucose in almost physiological ranges [22], they need implants with surgical placement [23], thus are not adequate for noninvasive monitoring. On the other hand, coherent Raman scattering methods were rarely studied for glucose monitoring (for instance, see [24]) and might be interesting to investigate in the light of newly emerged technologies, especially on the side of low noise detectors [25].
Although the first (spontaneous) Raman scattering acquisition of glucose solutions coupled with multivariate data analysis methods dates back to the 1980s and 1990s [21, 26], these studies either fail in demonstrating a strong relationship between concentration difference and Raman intensity or are often focused on higher glucose concentrations starting from $5 \mathrm{mmol} / \mathrm{l}$, so skipping lower glucose levels that are typically observed in hypoglycemia [27, 28]. This paper proposes a very selective approach based on exploiting a single Raman shift peak of $1125 \pm 10 \mathrm{~cm}^{-1}$ for non-enhanced Raman spectroscopy by sensing the lowest possible glucose levels typically seen in hypoglycemia. We validate the proposed sensing methodology on water-based glucose solutions with mixtures of lactate and urea, the two most common molecules found in human serum with similarities in their concentration, size, and molecule weight that usually interfere with Raman scattering based glucose detection. Our experimental results fill the literature gap by reporting glucose concentration measurements' sensitivity to demonstrate excellent performance in sensing physiopathological glucose levels, especially targeting low concentrations.

\section{Methodology}

\subsection{Sample Preparation}

The d-(+)-glucose, sodium 1-lactate, and urea powders were purchased from Sigma-Aldrich (MilliporeSigma, MO, USA). The human physiopathological glucose level could vary from 1 to $30 \mathrm{mmol} / \mathrm{l}$. Thus, a total of 13 samples of aqueous glucose solutions were prepared with a concentration of $1-5 \mathrm{mmol} / \mathrm{l}$, $5-10 \mathrm{mmol} / \mathrm{l}$, and $10-60$ with intervals of $1 \mathrm{mmol} / 1,2 \mathrm{mmol} / \mathrm{l}$, and $20 \mathrm{mmol} / \mathrm{l}$, respectively, and a single solution with 100 $\mathrm{mmol} / \mathrm{l}$. Additionally, lactate and urea aqueous solutions in the range of $1-200 \mathrm{mmol} / \mathrm{l}$ and a total of 36 solutions of lactate, urea, and glucose mixtures were prepared. The powder was carefully measured with a highly precise scale in each sample and then wholly dissolved in deionized water. Then, the solutions were stored overnight before measurement. During the measurement session, each sample was first stirred to ensure that the analyte was homogeneously dissolved, and a micropipette (Gilson International, France) was used to transfer a $20-\mu l$ droplet of each solution into the well of a concave microscope slide (Electron Microscopy Sciences, PA, USA).

\subsection{Data Acquisition and Analysis}

The Raman scattering spectrum of each sample solution was obtained with a backscattered confocal micro-Raman microscope (LabRAM HR, HORIBA, Japan) in a spectral region of $300 \mathrm{~cm}^{-1}$ to $2000 \mathrm{~cm}^{-1}$. Raman spectroscopy was employed with a $532-\mathrm{nm}$ green laser source set to $200 \mathrm{~mW}$ of power 
through the built-in neutral density filters. The filtered beam was focused using a $\times 50$ objective lens, and the confocal hole size was adjusted to $400 \mu \mathrm{m}$. Calibration of the spectrometer was carried out before the measurement sessions using the characteristic peak of silicon at $520 \mathrm{~cm}^{-1}$. The acquisition time for each scan was $120 \mathrm{~s}$ or $360 \mathrm{~s}$. Three consecutive spectra were obtained using different droplets to compute the measurement error. The room was dark, and a temperature of 24 ${ }^{\circ} \mathrm{C}$ was maintained throughout the experiments.

All data processing was performed with a data analysis tool (Origin, OriginLab Corporation, MA, USA). For each spectrum, the $950 \mathrm{~cm}^{-1}$ to $1200 \mathrm{~cm}^{-1}$ range was analyzed. First, the baseline spectrum was subtracted using the asymmetric least-square fit. Then, the Savitzky-Golay filter with polynomial order of three and a window length of thirteen was applied to smooth the signal and minimize the variation before peak intensity analysis and integration [29]. The absolute area under the Raman shift peaks of $861 \pm 10 \mathrm{~cm}^{-1}$ for lactate, $1005 \pm 15 \mathrm{~cm}^{-1}$ for urea, and $1125 \pm 10 \mathrm{~cm}^{-1}$ for glucose was chosen to construct the calibration curve for each analyte. Additionally, in the more extended 360-s acquisition time measurements, narrowband random cosmic rays peaks that inevitably appear in the spectra were removed on the spot [20].

\section{Results and Discussion}

\subsection{Sensitivity Investigation}

Figure 1a illustrates the raw Raman spectra of aqueous glucose solutions in various concentrations between $100 \mathrm{mmol} / \mathrm{l}$ and $1 \mathrm{mmol} / \mathrm{l}$ and water. It has been suggested that the intensity of inelastic scattering is directly proportional to the concentration of the solution [30]. This is observed at multiple peaks of the glucose spectra, typically around $437 \mathrm{~cm}^{-1}, 518$ $\mathrm{cm}^{-1}, 1060 \mathrm{~cm}^{-1}, 1125 \mathrm{~cm}^{-1}, 1365 \mathrm{~cm}^{-1}$, and $1461 \mathrm{~cm}^{-1}$ Raman shifts. However, the spectra area around $950 \mathrm{~cm}^{-1}$ to $1200 \mathrm{~cm}^{-1}$ seems extra sensitive to variations in lower concentrations. Thus, this range, the signature region, is optimum for glucose concentration measurements, which was exploited before to predict the glucose level [31]. The relationship between the amplitude of $1060 \mathrm{~cm}^{-1}$ and $1125 \mathrm{~cm}^{-1}$ Raman peaks with the glucose concentration change is illustrated in Fig. 1a zoom-in. The Raman shift peaks at $1060 \mathrm{~cm}^{-1}$ and $1125 \mathrm{~cm}^{-1}$ have been widely associated with the $\mathrm{CO}$ stretching and $\mathrm{COH}$ bonds' bending mode, respectively [21, 32]. Spectra after data processing (i.e., background removal and smoothing) in the signature region is presented in Fig. $1 \mathrm{~b}$.

Furthermore, Fig. 2a shows that the characteristic peak of $1125 \mathrm{~cm}^{-1}$ is sensitive enough to accurately sense the low glucose concentration values, while the region at lower wavenumbers around $1060 \mathrm{~cm}^{-1}$ is highly disrupted below 5 $\mathrm{mmol} / \mathrm{l}$. This observation is consistent with the glucose level studies of mice in the $5-15 \mathrm{mmol} / \mathrm{l}$ range [33]. In contrast, previous studies expressed the desire to use the entire (fingerprint) spectrum or larger areas of blood's Raman spectrum for glucose level prediction and, hence, inevitably proposed multivariate statistical data analysis techniques to improve the sensitivity [34-37].

This study thereby challenges the prior findings. We speculate that as the entire spectrum of glucose is not sensitive enough to predict lower ranges even in an aqueous solution, it is hardly possible to improve the measurement's sensitivity for in vivo studies. Therefore, applying univariate analysis was preferred by merely calculating the area under the curve at $1125 \pm 10 \mathrm{~cm}^{-1}$. Subsequently, the calibration curve was computed, and a linear relationship was obtained and shown in Fig. 2b. The linear fit with an $R^{2}$ value of 0.96 and $\sim 946$ counts/mM sensitivity was recorded.

More sensitive Raman spectra can be acquired by increasing either (or both) the source laser power and the acquisition time [38]. Thus, another set of experiments was conducted with an acquisition time of $360 \mathrm{~s}$. The output spectra after data processing are illustrated in Fig. 3a. Figure $3 \mathrm{~b}$ presents the calibration curve with an $R^{2}$ value of 0.97 and $\sim 4072$ counts/mM sensitivity. A quick visual comparison of spectra below $5 \mathrm{mmol} / \mathrm{l}$ of Fig. 2a with Fig. 3a and the absolute value
Fig.1 a Raw Raman scattering spectra of glucose powder dissolved in aqueous solutions in the range of $100 \mathrm{mmol} / \mathrm{l}$ to physiological ranges and the lowest physiopathological glucose levels; zoom-in the spectra in the signature region $950-1200 \mathrm{~cm}^{-1}$. b Processed Raman scattering spectra of aqueous glucose solutions in the signature region. The peaks indicate the glucose level increase as a function of concentration
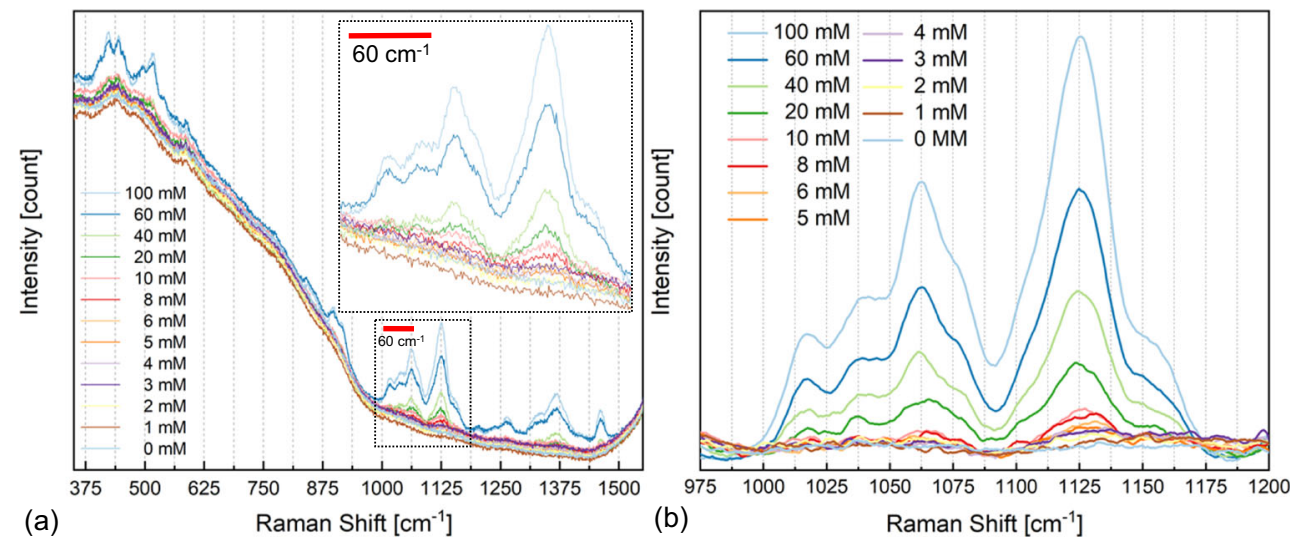
Fig. 2 a Processed Raman scattering spectra of aqueous glucose solutions in low concentrations in the range of 10 $\mathrm{mmol} / \mathrm{l}$ to water with an acquisition time of $120 \mathrm{~s}$; the characteristic peak under analysis is highlighted. b The calibration curve for glucose concentration prediction is computed by the area under the curve at $1125 \pm$ $10 \mathrm{~cm}^{-1}$; three consecutive measurements return the error bars
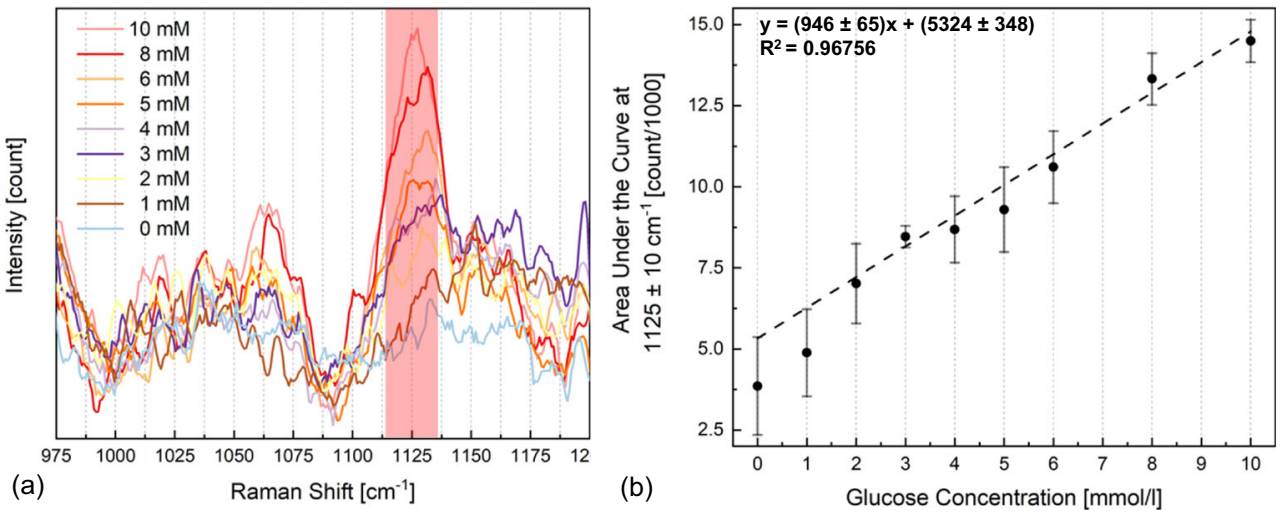

of the calibration curves' slope in Fig. $2 b$ and Fig. $3 b$ show that sensitivity is improved almost 4 times. Still, the area around $1125 \mathrm{~cm}^{-1}$ is a reliable choice for glucose concentration prediction in low levels reaching even to sub $1 \mathrm{mmol} / 1$ ranges (data not shown here) in contrast with the area around $1060 \mathrm{~cm}^{-1}$ the spectra disrupted below $3 \mathrm{mmol} / \mathrm{l}$.

\subsection{Selectivity Investigation}

Raman spectroscopy's selectivity is undoubtedly a challenge for accurate determination of the glucose level in native fluid form in the human body with interferences from other background molecules. The well-known method to circumvolve the interferences in the prior art is feeding each of the interference molecule's Raman spectrum (or at least the more intense ones) to multivariate data analysis algorithms to distinguish and remove their effects [39]. An immediate drawback of this method is the high dependency on calibration procedures. These procedures should be repeated multiple times during continuous monitoring sessions due to each molecule's variation upon different activities or generally due to differences in person-to-person physiologies [34]. However, if the area around the characteristic peak of glucose is used to predict its level, as proposed in this study, no potential intensive signature from other molecules would be present, and then the univariate analysis will offer inherent advantages. Thus, we further study the interference from other molecules to this peak. This investigation is also critical to ensure glucose detection's feasibility at low concentrations by the proposed method. For instance, urea's in vivo detection thought its Raman characteristic peak would be highly challenging since there is a clear intensive peak due to phenylalanine in that region. Phenylalanine is an amino acid found in hemoglobin with a well-known Raman spectrum [40].

Although previous studies of whole blood Raman spectrum suggested no other intensive effect of other molecules to characteristic glucose peak as similar to urea with phenylalanine case [41], a detailed study of other potentially interfering molecules with varying concentrations is still necessary. Here, lactate and urea, the two most similar molecules in concentration and size to glucose in human blood, were studied. Figure $4 a$ and $b$ illustrates the fingerprint region of the processed Raman spectra of aqueous lactate and urea solutions, respectively, with concentration varying from 0 to 200 $\mathrm{mmol} / \mathrm{l}$. Studying the sensitivity of various peaks on low concentration (Fig. 4 zoom-ins) shows that the Raman shift peak of $861 \pm 10 \mathrm{~cm}^{-1}$ could be acquired to predict lactate concentration, and similarly, the very Raman-sensitive characteristic peak of $1005 \pm 15 \mathrm{~cm}^{-1}$ could be used to predict urea concentration. These observations are persistent with previous findings [42]. Both lactate and urea show small peak(s) at their high concentration values in the signature region of glucose,
Fig. 3 a Processed Raman scattering spectra of aqueous glucose solutions with a longer acquisition time of $360 \mathrm{~s}$. b The calibration curve of glucose concentration versus area under the curve of $1125 \pm 10 \mathrm{~cm}^{-1}$. The sensitivity improved four times than the measurement with acquisition time of $120 \mathrm{~s}$
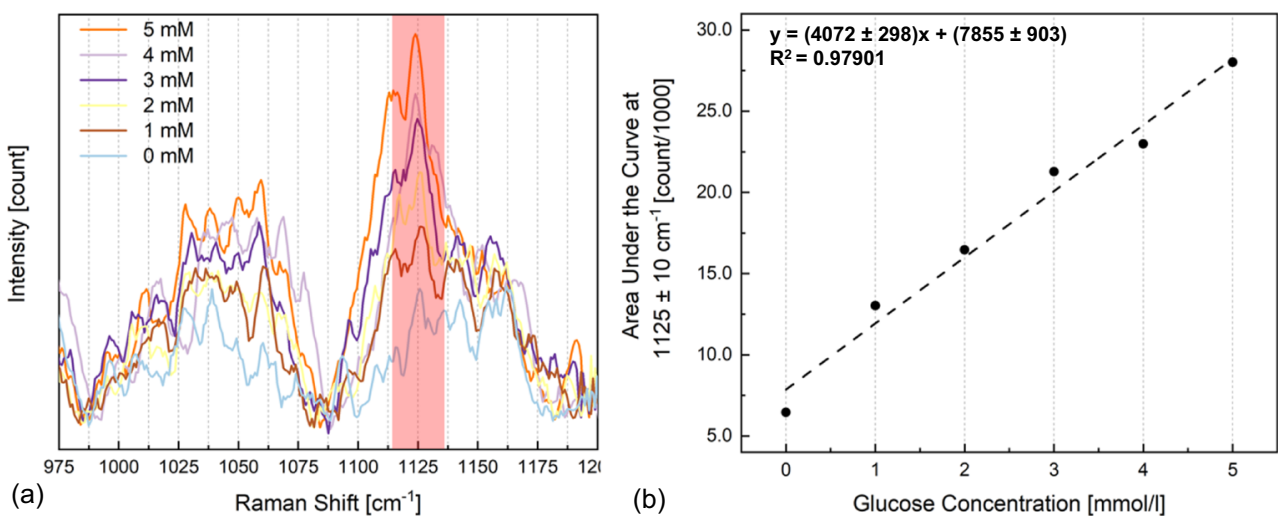
Fig. 4 a Processed Raman scattering spectra of aqueous lactate and $\mathbf{b}$ urea solutions. Zoom-in shows the spectra in the concentration range of $0-10$ $\mathrm{mmol} / \mathrm{l}$. The characteristic peaks of $861 \pm 10 \mathrm{~cm}^{-1}$ for lactate and $1005 \pm 15 \mathrm{~cm}^{-1}$ for urea are highlighted. The intensity difference between the characteristic peaks of lactate and urea is about 2 times, with urea being more intensive
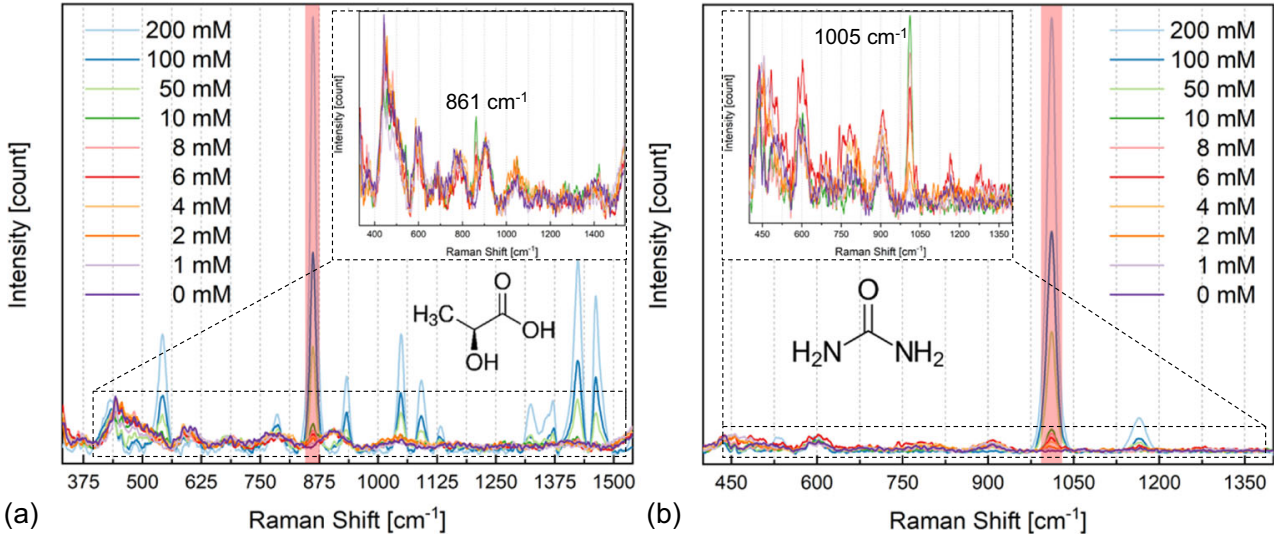

but their effect seems to minimize toward lower concentrations. For a comprehensive study, mixture solutions were prepared in various ranges to investigate the interference from lactate and urea variations on glucose. Figure 5 illustrates one of the prepared mixtures, where the superposition of lactate, urea, and glucose spectrum (dotted lines) creates the final mixture spectrum (solid line) [38]. Note that the intensity of the individual molecule solution Raman characteristic peaks is almost three times their mixture solution, which is expected since each analytes' concentration ratio in the mixture is $1: 3$.

Figure $6 \mathrm{a}$ and $\mathrm{b}$ illustrates the calibration curves acquired when the lactate and urea concentrations were varied in the range of 2-40 mmol/l, respectively, when other molecules' concentration was kept constant. A minimum of $2 \mathrm{mmol} / \mathrm{l}$ concentrations was selected instead of $1 \mathrm{mmol} / \mathrm{l}$ due to lactate's low sensitivity

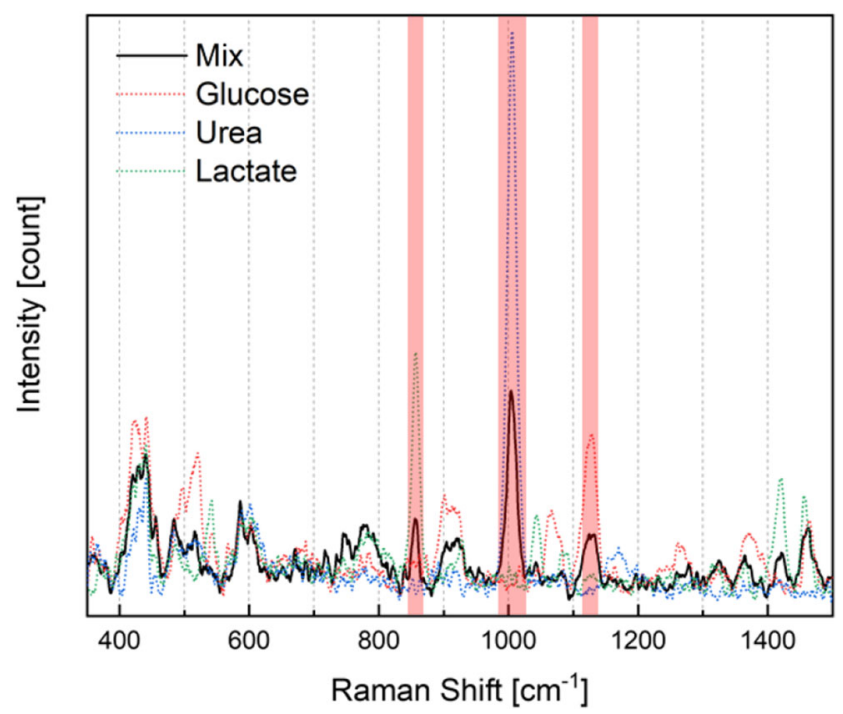

Fig. 5 Processed Raman scattering spectrum of lactate, urea, and glucose mixture aqueous solution (solid line) with a concentration of $10 \mathrm{mmol} / \mathrm{l}$ from each molecule prepared in $30-\mathrm{mL}$ solution and individual aqueous spectra of molecules in dotted lines with contraction of $30 \mathrm{mmol} / \mathrm{l}$ prepared in 10-mL solutions. Raman spectrum of the mixture consists of the superposition of the individual spectrum of each aqueous solution. The characteristic Raman peaks of molecules are highlighted below $2 \mathrm{mmol} / \mathrm{l}$. Two sets of mixture solutions were prepared for each experiment. In the first set, the concentration of glucose was $8 \mathrm{mmol} / \mathrm{l}$, and in the second, it was $4 \mathrm{mmol} / \mathrm{l}$. The calibration curves evidently show that the respective Raman intensity increases with the slope of $\sim 1075$ counts/mM when the lactate concentration is changing. However, there is almost no change in the glucose curves' slopes. A similar interpretation holds for urea; the Raman intensity increases with the concentration change, with the slope of $\sim 3920$ counts/mM, but the glucose curve is flat and even slightly negative. Therefore, these findings suggest no potential interference from lactate and urea to glucose when measured with the proposed method. The slight decrease in glucose intensity in Fig. 6b is considered the upper limit of glucose detection. That limitation was not observed with lactate change in Fig. 6a because urea's characteristic peak is more intensive than the lactate's characteristic peak. Additionally, the similar difference between glucose concentration predictions of two sets in two experiments independently certifies measurements' accuracy during the experiment's multiple days.

\section{Conclusion}

This study demonstrates a very selective detection of physiopathological glucose levels by spontaneous Raman spectroscopy in the lowest possible physiological glucose levels, as usually found in hypoglycemic patients' blood. Compared to previous studies, a more straightforward univariate quantitative data analysis approach targeting a very narrow band of the glucose Raman spectrum is used instead of multivariate analysis, focusing on the entire spectrum [26, 27, 43]. This study also investigated the proposed approach's high-selectivity by showing that the area under the $1125 \mathrm{~cm}^{-1}$ Raman shift band is the ideal choice for predicting low glucose concentrations. Our study also confirmed that, instead, the bands around 437 $\mathrm{cm}^{-1}, 1050 \mathrm{~cm}^{-1}$, and $1365 \mathrm{~cm}^{-1}$ are highly affected by other interfering metabolites, even with intensifying the scattering effect by a factor of three. Moreover, this study showed that the univariate analysis brings inherent advantages over 


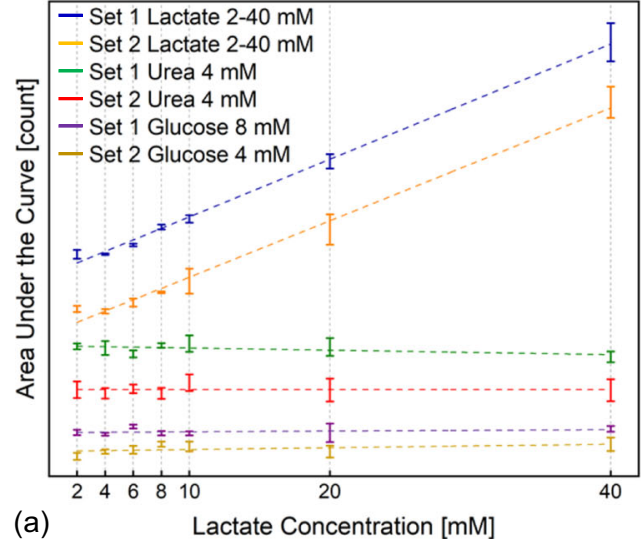

(a)

Lactate Concentration [mM]

Fig. 6 The calibration curves of lactate, urea, and glucose mixture solutions in two sets showing a lactate and $\mathbf{b}$ urea concentrations when varied versus area under the curves of their characteristic peaks (i.e., 861 $\pm 10 \mathrm{~cm}^{-1}$ for lactate, $1005 \pm 15 \mathrm{~cm}^{-1}$ for urea, and $1125 \pm 10 \mathrm{~cm}^{-1}$ for glucose). The positive calibration curve slopes of lactate $(\sim 1075$ counts/

multivariate analysis, given that the background molecules' effects in the blood's entire fingerprint region are not intensively present in the proposed band. Finally, this study presented lactate and urea sensing in their low concentration ranges using their characteristic Raman shift peaks of $861 \pm$ $10 \mathrm{~cm}^{-1}$ and $1005 \pm 15 \mathrm{~cm}^{-1}$, respectively. Our research provided the first essential steps of more comprehensive research by showing the feasibility of very selective glucose detection for low concentration levels. The present manuscript tackles the demonstration of the relevance of the univariate analysis for glucose sensing in the whole physiopathological range indeed. The next step toward a portable system will address two further main problems: first, the interference due to other blood components and, second, the ways to reduce the amount of optical power required to achieve an excellent signal-tonoise ratio. The future work is then planned to address those two further main issues to pave the way for future portable glucose sensing devices based on Raman scattering.

Acknowledgements The authors gratefully thank Dr. Richard Gaal for highly fruitful discussions about Raman spectroscopy.

Author's Contribution AG designed and conducted the experiments, performed the data analysis, and wrote the manuscript. TL helped in conducting experiments. $\mathrm{AB}, \mathrm{AC}$, and $\mathrm{SC}$ reviewed the manuscript. $\mathrm{SC}$ conceived the experiments. $\mathrm{CE}$ and $\mathrm{SC}$ supervised the project.

Funding Open Access funding provided by EPFL Lausanne. This work was supported by the École Polytechnique Fédérale de Lausanne (EPFL) research fund.

Availability of Data and Material Available upon request from the corresponding author.

Code Availability Available upon request from the corresponding author.

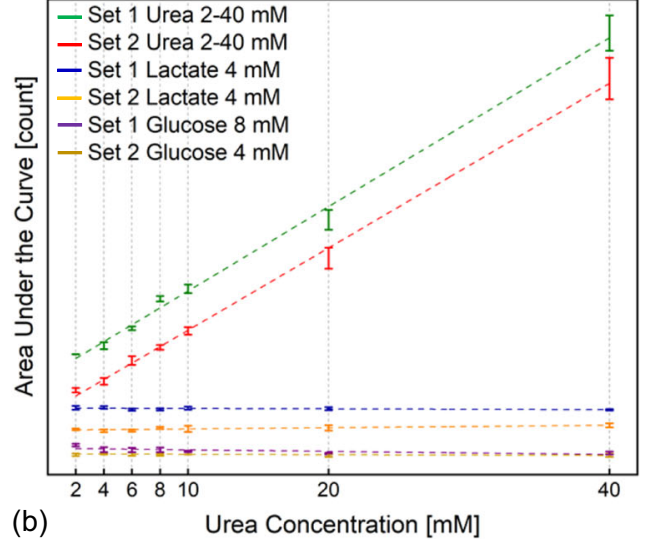

$\mathrm{mM})$ and urea $(\sim 3920$ counts $/ \mathrm{mM})$ versus the flat curve of glucose suggest no potential interference from lactate and urea to glucose when measured with the proposed method. For clarity, offsets are added intentionally to all curves but the glucose. Three consecutive measurements return the error bars

\section{Declarations}

Conflict of Interest None.

Research Involving Humans and Animals Statement None.

Informed Consent None.

Open Access This article is licensed under a Creative Commons Attribution 4.0 International License, which permits use, sharing, adaptation, distribution and reproduction in any medium or format, as long as you give appropriate credit to the original author(s) and the source, provide a link to the Creative Commons licence, and indicate if changes were made. The images or other third party material in this article are included in the article's Creative Commons licence, unless indicated otherwise in a credit line to the material. If material is not included in the article's Creative Commons licence and your intended use is not permitted by statutory regulation or exceeds the permitted use, you will need to obtain permission directly from the copyright holder. To view a copy of this licence, visit http://creativecommons.org/licenses/by/4.0/.

\section{References}

1. Zimmet, P. (2001). Globalization, coca-colonization and the chronic disease epidemic: Can the Doomsday scenario be averted? Journal of Internal Medicine, 249(S741), 17-26.

2. World Health Organization. Available from https://www.who.int/ health-topics/diabetes\#tab=tab_1. Accessed 21st of November, 2020.

3. Zimmet, P., Alberti, K., \& Shaw, J. (2001). Global and societal implications of the diabetes epidemic. Nature, 414(6865), 782-787.

4. Garg, S. K., Rewers, A. H., \& Akturk, H. K. (2018). Ever-increasing insulin-requiring patients globally. Mary Ann Liebert, Inc.

5. Trief, P. M., Cibula, D., Rodriguez, E., Akel, B., \& Weinstock, R. S. (2016). Incorrect insulin administration: A problem that warrants attention. Clinical Diabetes, 34(1), 25-33.

6. Polonsky, W. H., \& Fisher, L. (2013). Self-monitoring of blood glucose in noninsulin-using type 2 diabetic patients: Right answer, but 
wrong question: Self-monitoring of blood glucose can be clinically valuable for non-insulin users. Diabetes Care, 36(1), 179-182.

7. Negrato, C. A., \& Zajdenverg, L. (2012). Self-monitoring of blood glucose during pregnancy: Indications and limitations. Diabetology $\&$ Metabolic Syndrome, 4(1), 54.

8. Barman, I., et al. (2012). Raman spectroscopy-based sensitive and specific detection of glycated hemoglobin. Analytical Chemistry, 84, 2474-2482.

9. Pandey, R., et al. (2015). Emerging trends in optical sensing of glycemic markers for diabetes monitoring. TrAC Trends in Analytical Chemistry, 64, 100-108.

10. Hughes, M. D. (2009). The business of self-monitoring of blood glucose: A market profile. Journal of Diabetes Science and Technology, 3(5), 1219-1223.

11. Olansky, L., \& Kennedy, L. (2010). Finger-stick glucose monitoring: Issues of accuracy and specificity. American Diabetes Association, 33, 948-949.

12. Heinemann, L. (2008). Finger pricking and pain: A never ending story. Journal of Diabetes Science and Technology, 2(5), 919-921.

13. Cradock, S., \& Hawthorn, J. (2002). Pain, distress and blood glucose monitoring. Journal of Diabetes Nursing, 6(6), 188-191.

14. Abdoli, S., Hessler, D., Vora, A., Smither, B., \& Stuckey, H. (2020). Descriptions of diabetes burnout from individuals with Type 1 diabetes: An analysis of YouTube videos. Diabetic Medicine, 37(8), 1344-1351.

15. Wang, J. (2008). Electrochemical glucose biosensors. Chemical Reviews, 108(2), 814-825.

16. Vaddiraju, S., Burgess, D. J., Tomazos, I., Jain, F. C., \& Papadimitrakopoulos, F. (2010). Technologies for continuous glucose monitoring: Current problems and future promises. Journal of Diabetes Science and Technology, 4(6), 1540-1562.

17. Chetty, V., Almulla, A., Odueyungbo, A., \& Thabane, L. (2008). The effect of continuous subcutaneous glucose monitoring (CGMS) versus intermittent whole blood finger-stick glucose monitoring (SBGM) on hemoglobin A1c (HBA1c) levels in Type I diabetic patients: A systematic review. Diabetes Research and Clinical Practice, 81(1), 79-87.

18. Vashist, S. K. (2012). Non-invasive glucose monitoring technology in diabetes management: A review. Analytica Chimica Acta, 750, $16-27$.

19. Losoya-Leal, A., Camacho-León, S., Dieck-Assad, G., \& MartínezChapa, S. O. (2012). State of the art and new perspectives in noninvasive glucose sensors. Revista Mexicana De Ingeniería Biomédica, 33(1), 41-52.

20. Baker, M. J., Hughes, C. S., \& Hollywood, K. A. (2016). Biophotonics: Vibrational spectroscopic diagnostics. Morgan \& Claypool Publishers.

21. Mathlouthi, M., \& Luu, D. V. (1980). Laser-Raman spectra of dglucose and sucrose in aqueous solution. Carbohydrate Research, 81(2), 203-212.

22. Lyandres, O., et al. (2008). Progress toward an in vivo surfaceenhanced Raman spectroscopy glucose sensor. Diabetes Technology \& Therapeutics, 10(4), 257-265.

23. Stuart, D. A., et al. (2006). In vivo glucose measurement by surfaceenhanced Raman spectroscopy. Analytical Chemistry, 78(20), $7211-7215$.

24. R. V. Tarr, and P. G. Steffes (1993). "Non-invasive blood glucose measurement system and method using stimulated Raman spectroscopy," Google Patents.

25. Boukhayma, A. (2018). "Low-noise CMOS image sensors," Ultra Low Noise CMOS Image Sensors (pp. 13-34). Springer.
26. Wang, S. Y., et al. (1993). Analysis of metabolites in aqueous solutions by using laser Raman spectroscopy. Applied Optics, 32(6), 925-929.

27. Shih, W.-C., Bechtel, K. L., \& Rebec, M. V. (2015). Non-invasive glucose sensing by transcutaneous Raman spectroscopy. Journal of Biomedical Optics, 20, 051036.

28. Berger, A. J., et al. (1999). Multicomponent blood analysis by nearinfrared Raman spectroscopy. Applied Optics, 38, 13.

29. A. R. M. Radzol, Y. L. Khuan, W. Mansor, and A. Azman. "Optimization of Savitzky-Golay smoothing filter for salivary surface enhanced Raman spectra of non structural protein 1." TENCON 2014-2014 IEEE Region 10 Conference. IEEE, 2014.

30. Do Nascimento, G. M. (2018). Raman Spectroscopy. IntechOpen, pp 3-10.

31. Xu, Y., Ford, J. F., Mann, C. K., Vickers, T. J., Brackett, J. M., Cousineau, K. L., \& Robey, W. G. (1997). Raman measurement of glucose in bioreactor materials. In Biomedical Sensing, Imaging, And Tracking Technologies Ii 2976, 10-19.

32. Dudek, M., et al. (2019). Raman optical activity and Raman spectroscopy of carbohydrates in solution. Spectrochimica Acta Part A: Molecular and Biomolecular Spectroscopy, 206, 597-612.

33. Shao, J., Lin, M., Li, Y., et al. (2012). In vivo blood glucose quantification using Raman spectroscopy. PLoS One, 7(10), e48127.

34. Lipson, J., Bernhardt, J., Block, U., et al. (2009). Requirements for calibration in non-invasive glucose monitoring by Raman spectroscopy. SAGE Publications.

35. Singh, S. P., Mukherjee, S., Galindo, L. H., et al. (2018). Evaluation of accuracy dependence of Raman spectroscopic models on the ratio of calibration and validation points for non-invasive glucose sensing. Analytical and Bioanalytical Chemistry, 410(25), 64696475.

36. Enejder, A. M., Scecina, T. G., Oh, J., et al. (2005). Raman spectroscopy for non-invasive glucose measurements. Journal of Biomedical Optics, 10(3), 031114.

37. Dingari, N. C., Barman, I., Kang, J. W., et al. (2011). Wavelength selection-based nonlinear calibration for transcutaneous blood glucose sensing using Raman spectroscopy. Journal of Biomedical Optics, 16(8), 087009.

38. Braun, F., et al. (2016). Highly sensitive Raman spectroscopy with low laser power for fast in-line reaction and multiphase flow monitoring. Analytical Chemistry, 88, 9368-9374.

39. Ren, M., \& Arnold, M. A. (2007). Comparison of multivariate calibration models for glucose, urea, and lactate from nearinfrared and Raman spectra. Analytical and Bioanalytical Chemistry, 387(3), 879-888.

40. Liu, S.-Y., Tian, X.-D., Zhang, Y., et al. (2018). Quantitative surface-enhanced Raman spectroscopy through the interfaceassisted self-assembly of three-dimensional silver nanorod substrates. Analytical Chemistry, 90(12), 7275-7282.

41. Enejder, A. M., Koo, T.-W., Oh, J., et al. (2002). Blood analysis by Raman spectroscopy. Optics Letters, 27(22), 2004-2006.

42. Wascotte, V., et al. (2007). Assessment of the "skin reservoir" of urea by confocal Raman microspectroscopy and reverse iontophoresis in vivo. Pharmaceutical Research, 24(10), 1897-1901.

43. Byrne, H. J., Bonnier, F., McIntyre, J., \& Parachalil, D. R. (2020). Quantitative analysis of human blood serum using vibrational spectroscopy. Clinical Spectroscopy, 2, 100004.

Publisher's Note Springer Nature remains neutral with regard to jurisdictional claims in published maps and institutional affiliations. 